\title{
Dynamic Spike Thresholds during Synaptic Integration Preserve and Enhance Temporal Response Properties in the Avian Cochlear Nucleus
}

\author{
MacKenzie A. Howard and Edwin W Rubel \\ Department of Physiology and Biophysics and Virginia Merrill Bloedel Hearing Research Center, University of Washington, Seattle, Washington 98195
}

\begin{abstract}
Neurons of the cochlear nuclei are anatomically and physiologically specialized to optimally encode temporal and spectral information about sound stimuli, in part for binaural auditory processing. The avian cochlear nucleus magnocellularis (NM) integrates excitatory eighth nerve inputs and depolarizing GABAergic inhibition such that temporal fidelity is enhanced across the synapse. The biophysical mechanisms of this depolarizing inhibition, and its role in temporal processing, are not fully understood. We used whole-cell electrophysiology and computational modeling to examine how subthreshold excitatory inputs are integrated and how depolarizing IPSPs affect spike thresholds and synaptic integration by chick NM neurons. We found that both depolarizing inhibition and subthreshold excitatory inputs cause voltage threshold accommodation, nonlinear temporal summation, and shunting. Inhibition caused such large changes in threshold that subthreshold excitatory inputs were followed by a refractory period. We hypothesize that these large shifts in threshold eliminate spikes to asynchronous inputs, providing a mechanism for the enhanced temporal fidelity seen across the eighth nerve/ cochlear nucleus synapse. Thus, depolarizing inhibition and threshold shifting hone the temporal response properties of this system so as to enhance the temporal fidelity that is essential for auditory perception.
\end{abstract}

\section{Introduction}

Action potential timing is an important factor in neural processing, particularly in the auditory system in which the temporal fine structure of a sequence of spikes can directly represent the frequency and phase of sound stimuli (Oertel, 1999). Sound frequency and phase are essential for binaural hearing and sound localization.

Many lower auditory neurons exhibit hallmark specializations for high-frequency firing and maximal temporal fidelity (e.g., large voltage-activated $\mathrm{K}^{+}$currents, low input resistance, fast time constant, high spike threshold, small spike half-width) (Reyes et al., 1994; Zhang and Trussell, 1994a,b; Rathouz and Trussell, 1998; Scott et al., 2005). Depression and desensitization of excitatory synapses improve the fidelity between inputs and outputs (Brenowitz and Trussell, 2001; Kuba et al., 2002; Cook et al., 2003). Axonal spike generation in mouse spiral ganglion and chicken nucleus magnocellularis (NM) and laminaris (NL) also improves the temporal accuracy of spike outputs (Hossain et al., 2005; Kuba et al., 2006; Kuba and Ohmori, 2009). Cochlear nucleus bushy cells have diminutive dendritic arbors, which de-

Received April 11, 2010; revised June 24, 2010; accepted July 18, 2010.

This work was supported by funding from National Institutes for Deafness and Other Communication Disorders Grants DC03829, DC04661, and DC08035. We gratefully acknowledge the assistance of Sean Slee, Matthew Higgs, William Spain, Adrienne Fairhall, Brian Lundstrom, Rebecca Mease, and Sungho Hong for their invaluable help with modeling. We also thank George Pollak, Josh Gittleman, Audrey Brumback, R. Michael Burger, Yong Lu, Leonard Kitzes, and Jane Sullivan for insights in the preparation of this manuscript.

Correspondence should be addressed to Dr. Edwin W Rubel, Virginia Merrill Bloedel Hearing Research Center, University of Washington, Box 357923, Seattle, WA 98195. E-mail: rubel@u.washington.edu.

DOI:10.1523/JNEUROSCI.1840-10.2010

Copyright $\odot 2010$ the authors $\quad 0270-6474 / 10 / 3012063-12 \$ 15.00 / 0$ crease dendritic filtering (Jhaveri and Morest, 1982). Mature chick NM and NL neurons exhibit depolarizing GABAergic responses (Hyson et al., 1995; Lu and Trussell, 2001; Monsivais and Rubel, 2001; Tang et al., 2009). This depolarizing inhibition makes EPSPs smaller and briefer, and may improve timing in this system by decreasing "coincidence detection window" duration, when excitatory synaptic inputs can temporally integrate (Funabiki et al., 1998; Yang et al., 1999), and balancing excitation between inputs from the two ears (Burger et al., 2005; Dasika et al., 2005).

$\mathrm{NM}$ and the homologous mammalian anteroventral cochlear nucleus receive excitation solely from a small number (two to four for NM) of eighth nerve axons (Jhaveri and Morest, 1982). In the past, these neurons were sometimes assumed to simply relay inputs in a one-to-one fashion to higher order nuclei. However, closer examination of spike activity reveals improved spike timing and lower firing rates in NM compared with eighth nerve axons (Fukui et al., 2006). We hypothesized that the synaptic and intrinsic properties of these neurons act to exclude asynchronous inputs (i.e., outside the coincidence detection window), thus improving the temporal fidelity of the auditory system. We used in vitro electrophysiology and computational modeling to examine the biophysical mechanisms of spike threshold dynamics during integration of excitatory and inhibitory input in NM. We show that spike threshold is very flexible in these neurons, particularly because of $\mathrm{Na}^{+}$channel inactivation and $\mathrm{K}^{+}$channel activation during depolarizing inhibition and subthreshold excitation. We also show that elevated firing rates, as during high levels of sound stimulation, synaptic depression and inhibition cause eighth nerve EPSPs to become subthreshold. Thus, NM neurons require 
multiple coincident inputs to reach spike threshold. During integration of excitatory and inhibitory input, the window for temporal integration is shortened and strong accommodation causes neurons to become briefly refractory after subthreshold input. This eliminates spiking in response to asynchronous inputs, which may provide a mechanism for improving phase locking across a broad dynamic range of sound intensities.

\section{Materials and Methods}

Ethical approval. The University of Washington Institutional Animal Care and Use Committee approved all procedures and experimental protocols used in this study.

Brainstem slice preparation. Thirty-four male and female White Leghorn chicks (Gallus domesticus) were used in this study. Fertilized eggs were purchased from Hy-line and incubated and hatched on site. At posthatch day 2 (P2) to P3, chicks were quickly decapitated and the whole brainstem was removed into an oxygenated ice-cold slicing solution, containing the following (in $\mathrm{mM}$ ): $130 \mathrm{NaCl}, 3 \mathrm{KCl}, 1.25 \mathrm{NaH}_{2} \mathrm{PO}_{4}, 26$ $\mathrm{NaHCO}_{3}, 1 \mathrm{MgCl}_{2}, 2 \mathrm{CaCl}_{2}$, and 10 glucose, $\mathrm{pH} \mathrm{7.4;} \mathrm{osmolarity,} 300$ $\mathrm{mOsm} / \mathrm{L}$. The brainstem was blocked both rostral and caudal to the cochlear nuclei and glued into a vibratome slicing chamber (Technical Products International). The chamber was then filled with ice-cold slicing solution. The brainstem was sectioned coronally into slices $200 \mu \mathrm{m}$ thick. Sections containing NM were distinguishable by eye because of the large tract of fibers crossing the midline. These sections were placed in an incubating chamber containing slicing solution maintained at room temperature for a period of $1 \mathrm{~h}$.

After the incubation, selected slices were removed to a recording chamber mounted on the stage of a Zeiss Axioskop FS. This recording chamber was constantly superfused with warmed, oxygenated artificial CSF (ACSF). All experiments were performed at $35-36^{\circ} \mathrm{C}$.

Previous studies have determined that the physiological properties of NM neurons vary systematically along the tonotopic axis of NM (Fukui and Ohmori, 2004). Additionally, this axis exhibits a developmental gradient, with neurons responding to high characteristic frequencies reaching maturity more quickly than lower frequency neurons (Rubel et al., 1976; Jackson et al., 1982). To eliminate a bias in our data caused by tonotopic variation, we recorded from NM neurons throughout the tonotopic extent of NM and averaged across the nucleus. Examination of the data from individual neurons revealed tonotopic differences in some physiological measures (data not shown), following a similar pattern to that described by Fukui and Ohmori (2004). This tonotopic variation is reflected by the amplitude of the SD error bars illustrated in many figures of this report. However, the fundamental nature of the response properties that we report did not differ as a function of the tonotopic position of a NM neuron.

In vitro electrophysiology. For these experiments, the extracellular solution was ACSF, containing the following (in $\mathrm{mm}$ ): $130 \mathrm{NaCl}, 3 \mathrm{KCl}, 1.25$ $\mathrm{NaH}_{2} \mathrm{PO}_{4}, 26 \mathrm{NaHCO}_{3}, 1 \mathrm{MgCl}_{2}, 3 \mathrm{CaCl}_{2}$, and 10 glucose, pH 7.4; osmolarity, $300 \mathrm{mOsm} / \mathrm{L}$. We used a $\mathrm{K}^{+}$-based pipette solution for these experiments, containing the following (in $\mathrm{mM}$ ): $105 \mathrm{~K}$-gluconate, $35 \mathrm{KCl}$, $1 \mathrm{MgCl}_{2}, 10$ HEPES-K ${ }^{+}$, 5 EGTA, 4 ATP-Mg ${ }^{2+}$, and 0.3 GTP-Na ${ }^{+}$. All chemicals were obtained from Sigma-Aldrich.

Patch pipettes were pulled to resistances of 4-5 M 2 . Pipettes were filled with the appropriate solution and visually guided to NM using a $40 \times$ water-immersion objective and infrared-differential interference contrast optics. Once gigaohm seals between the pipette and the neuron were attained, the membrane patch was ruptured to establish the wholecell recording configuration. Neurons in which a gigaohm seal was not established, in which the membrane patch ruptured spontaneously, or that exhibited a resting membrane potential positive to $-60 \mathrm{mV}$ were excluded from analyses. Junction potentials for all experiments were calculated and corrected for.

Current-clamp experiments were performed using an Axoclamp 2B amplifier (Molecular Devices). All experiments were controlled with a Macintosh G4. Protocols were written and run with Axograph, version 4.5 , software (Molecular Devices). Data were low-pass filtered at $20 \mathrm{kHz}$ and digitized with an ITC-16 (InstruTECH) at 20 or $100 \mathrm{kHz}$.

\section{Table 1. Model current parameters}

\begin{tabular}{ll}
\hline$I_{\text {Na }}$ & $1 /(1+\exp (-(v+31) / 7.5)$ \\
$m_{\infty}$ & $1 /(1+\exp ((v+65) / 6.5)$ \\
$h_{\infty}$ & $\left(10 /\left(5^{*} \exp ((v+60) / 18)+36^{*} \exp (-(v+60) / 25)\right)+0.04\right.$ \\
$\tau_{\mathrm{m}}$ & $\left(100 /\left(7^{*} \exp ((v+60) / 11)+10^{*} \exp (-(v+60) / 25)\right)+0.6\right.$ \\
$\tau_{\mathrm{h}}$ & 3 \\
$\mathrm{q} 10$ & $22^{\circ} \mathrm{C}$ \\
$\mathrm{T}_{0}$ & \\
& \\
$I_{\mathrm{KL}}$ & $1 /(1+\exp (-(v+60.5) / 6)$ \\
$W_{\infty}$ & $1 /(1+\exp (-(v+71) / 10)$ \\
$z_{\infty}$ & $\left(100 /\left(6^{*} \exp ((v+60) / 6)+16^{*} \exp (-(v+60) / 45)\right)+1.5\right.$ \\
$\tau_{\mathrm{w}}$ & $(100 /(\exp ((v+60) / 20)+\exp (-(v+60) / 8))+50$ \\
$\tau_{\mathrm{z}}$ & 3 \\
$\mathrm{q} 10$ & $22^{\circ} \mathrm{C}$ \\
$\mathrm{T}_{0}$ & \\
& \\
$I_{\mathrm{KH}}$ & $1 /(1+\exp (-(v+42.5) / 4.5)$ \\
$n_{\infty}$ & $1 /(1+\exp (-(v+11.2) / 6)$ \\
$p_{\infty}$ & $\left(100 /\left(11^{*} \exp ((v+60) / 24)+21^{*} \exp (-(v+60) / 23)\right)+0.7\right.$ \\
$\tau_{\mathrm{n}}$ & $\left(100 /\left(4^{*} \exp ((v+60) / 32)+5^{*} \exp (-(v+60) / 22)\right)+5\right.$ \\
$\tau_{\mathrm{p}}$ & 3 \\
$\mathrm{q} 10$ & $22^{\circ} \mathrm{C}$ \\
$\mathrm{T}_{0}$ & \\
\hline
\end{tabular}

Conductance clamp experiments were performed using the same experimental devices with the addition of custom-built multiplying and summing amplifiers (Department of Physiology and Biophysics Electronics Shop, University of Washington, Seattle, WA). The multiplying amplifier received two inputs from the Axoclamp 2B. The first was from one of the output channels that sent current commands to the headstage and the neuron being recorded from. The second input was voltage information, the membrane potential of the neuron. The multiplying amplifier changed the amplitude of the current command based on the changing membrane potential of the neuron and the manually selected reversal potential ( $-37 \mathrm{mV}$ for inhibition in this system). We selected a range of 25-100 nS for our inhibitory conductances. This choice was based on two factors. The first was calculation of GABAergic conductances based on IPSC amplitudes reported by Lu and Trussell (2001), and our own previous work (Howard et al., 2007). Depending on stimulation intensity and frequency, data from these reports indicate that GABAergic conductance can range from 10 to $>200 \mathrm{nS}$. In preliminary experiments, our conductance clamp stimuli depolarized neurons from 8 to $20 \mathrm{mV}$, similar to depolarizing IPSPs evoked by synaptic stimulation. Thus, we believe the use of 25-100 nS of inhibitory conductance was within the physiological range of NM neurons.

The drawback of using this method for conductance clamp experiments is that it reduces the amplitude of the current command output signal. NM neurons have a very high threshold, and under conditions of depolarizing inhibition our amplifier could not produce stimuli large enough to reach threshold. Thus, for our purposes, conductance clamp was useful for producing inhibitory conductances but could not produce large enough excitatory conductances to reach threshold under many of our experimental conditions. Because of this limitation, we chose to use current clamp to mimic excitatory inputs for all of our in vitro experiments and use our multicompartment model when mimicking excitatory conductances.

Computational modeling. A single-compartment computational model was produced using NEURON 6.0. This compartment represented a spherical neuron $20 \mu \mathrm{m}$ in diameter. This model contained a high voltageactivated $\mathrm{K}^{+}$current $\left(I_{\mathrm{KL}}\right)$, a low voltage-activated $\mathrm{K}^{+}$current $\left(I_{\mathrm{KH}}\right)$, a Hodgkin-Huxley-type $\mathrm{Na}^{+}$current $\left(I_{\mathrm{Na}}\right)$, and a passive leak current. Equations for the voltage dependence and kinetics of gating of these currents are presented in Table 1 . The activation and inactivation kinetics and the half-maximal activation potential $\left(V_{1 / 2}\right)$ and slope factor $\left(K_{0}\right)$ of the activation and inactivation curves of the $I_{\mathrm{KL}}$ and $I_{\mathrm{KH}}$ were based on previously published analyses of $\mathrm{K}^{+}$currents in cochlear nucleus auditory neurons (Rathouz and Trussell, 1998; Rothman and Manis, 2003). 
Table 2. Single-compartment model parameters

\begin{tabular}{lc}
\hline Axial resistance & $50 \Omega \mathrm{cm}$ \\
Temperature & $35^{\circ} \mathrm{C}$ \\
$E_{\mathrm{Na}}$ & $50 \mathrm{mV}$ \\
$E_{\mathrm{K}}$ & $-80 \mathrm{mV}$ \\
$E_{\mathrm{Cl}}$ & $-37 \mathrm{mV}$ \\
Length & $20 \mu \mathrm{m}$ \\
Diameter & $20 \mu \mathrm{m}$ \\
$g_{\text {leak }}$ & $0.002 \mathrm{~S} / \mathrm{cm}^{2}$ \\
$g_{\mathrm{Na}}$ & $0.1 \mathrm{~S} / \mathrm{cm}^{2}$ \\
$g_{\mathrm{LVA}}$ & $0.006 \mathrm{~S} / \mathrm{cm}^{2}$ \\
$g_{\mathrm{HVA}}$ & $0.002 \mathrm{~S} / \mathrm{cm}^{2}$ \\
\hline
\end{tabular}

The $V_{1 / 2}, K_{0}$, and conductance of the $\mathrm{K}^{+}$and leak currents were adjusted such that the voltage-current function of the model replicated that of the empirical data recorded from P2-P3 NM neurons. The kinetics and voltage dependence of $I_{\mathrm{Na}}$ gating were the same as those used by Rothman and Manis (2003) for their model of mammalian ventral cochlear nucleus, originally based on work by Belluzzi and colleagues (1985) and Costa (1996). These variables were also adjusted for $I_{\mathrm{Na}}$ such that spike threshold and amplitude were similar between the model and the empirical data. The parameters for the single-compartment model are listed in Table 2.

A multicompartment model of NM was also produced using NEURON 6.0. This model is based primarily on a multicompartment model of NL neurons (Kuba et al., 2006). The sections consisted of a soma, an initial axon segment, a spike initiation zone, and 10 alternating myelinated internode axon segments and unmyelinated nodes of Ranvier. Each compartment contained $I_{\mathrm{KH}}, I_{\mathrm{KL}}, I_{\mathrm{Na}}$, and leak currents of different total conductances. As with the single-compartment model, $I_{\mathrm{KH}}, I_{\mathrm{KL}}$, and leak currents were adjusted such that the voltage-current function of the model matched the empirical data. $I_{\mathrm{Na}}$ was also adjusted such that the threshold and peak amplitude of spikes evoked from rest were similar to empirical data. The parameters for the multicompartment model are listed in Table 3.

Data analysis and statistics. Data were analyzed Matlab, version 7.2, Microsoft Excel 2004, and StatView, version 5.0. For statistical analyses, ANOVAs and $t$ tests were used where appropriate. All figures illustrate mean \pm SD or SEM as indicated in figure legends. The threshold for statistical significance was considered to be $p<0.05$.

\section{Results}

\section{Threshold changes in NM neurons}

The basic firing features of NM neurons in response to simple current-clamp stimuli have been well documented. Briefly, depolarizing current steps evoke a single action potential, followed by a steady-state membrane potential (Fig. 1A). Voltage-current $(V-I)$ functions of this steady state potential exhibit a highly nonlinear section within the first $15-20 \mathrm{mV}$ of depolarization, followed by a relatively linear section (Fig. $1 B$, open symbols). This can also be seen when input resistance is plotted as a function of membrane potential (Fig. 1C). Previous studies have ascribed the nonlinearity of the $V-I$ function to activation of the large low voltage-activated $\mathrm{K}^{+}$conductances exhibited by NM neurons (Reyes et al., 1994; Rathouz and Trussell, 1998).

Although these large depolarizing pulses evoke spikes in NM neurons in vitro, NM neurons receive two different types of depolarizing input in vivo. In addition to large depolarizing excitatory inputs from eight nerve fibers, NM neurons receive GABAergic projections from the SON, which are depolarizing but inhibitory (Monsivais et al., 2000; Lu and Trussell, 2001). Three biophysical mechanisms are thought to underlie this depolarizing inhibition: shunting (reduction of input resistance) by activation of $\mathrm{GABA}_{\mathrm{A}}$ receptors, shunting by activation of voltage-gated $\mathrm{K}^{+}$channels, and accommodation (depolarization
Table 3. Multicompartment model parameters

\begin{tabular}{|c|c|}
\hline \multicolumn{2}{|l|}{ Global parameters } \\
\hline Axial resistance & $50 \Omega \mathrm{cm}$ \\
\hline Temperature & $35^{\circ} \mathrm{C}$ \\
\hline$E_{\mathrm{Na}}$ & $50 \mathrm{mV}$ \\
\hline$E_{\mathrm{K}}$ & $-80 \mathrm{mV}$ \\
\hline$E_{\mathrm{Cl}}$ & $-37 \mathrm{mV}$ \\
\hline \multicolumn{2}{|l|}{ Soma } \\
\hline Length & $20 \mu \mathrm{m}$ \\
\hline Diameter & $20 \mu \mathrm{m}$ \\
\hline$g_{\text {leak }}$ & $0.002 \mathrm{~S} / \mathrm{cm}^{2}$ \\
\hline$g_{\mathrm{LVA}}$ & $0.0012 \mathrm{~S} / \mathrm{cm}^{2}$ \\
\hline$g_{\mathrm{HVA}}$ & $0.0003 \mathrm{~S} / \mathrm{cm}^{2}$ \\
\hline \multicolumn{2}{|l|}{ Axon initial segment } \\
\hline Length & $50 \mu \mathrm{m}$ \\
\hline Diameter & $2 \mu \mathrm{m}$ \\
\hline$g_{\text {leak }}$ & $0.000001 \mathrm{~S} / \mathrm{cm}^{2}$ \\
\hline \multicolumn{2}{|l|}{ Spike initiation zone } \\
\hline Length & $15 \mu \mathrm{m}$ \\
\hline Diameter & $1.3 \mu \mathrm{m}$ \\
\hline$g_{\text {leak }}$ & $0.0006 \mathrm{~S} / \mathrm{cm}^{2}$ \\
\hline$g_{\mathrm{Na}}$ & $23 \mathrm{~S} / \mathrm{cm}^{2}$ \\
\hline$g_{\mathrm{LVA}}$ & $0.4 \mathrm{~S} / \mathrm{cm}^{2}$ \\
\hline$g_{\mathrm{HVA}}$ & $0.9 \mathrm{~S} / \mathrm{cm}^{2}$ \\
\hline \multicolumn{2}{|c|}{ Internodal axon segments } \\
\hline Length & $80 \mu \mathrm{m}$ \\
\hline Diameter & $1.3 \mu \mathrm{m}$ \\
\hline$g_{\text {leak }}$ & $0.000001 \mathrm{~S} / \mathrm{cm}^{2}$ \\
\hline \multicolumn{2}{|l|}{ Nodes of Ranvier } \\
\hline Length & $2 \mu \mathrm{m}$ \\
\hline Diameter & $1.3 \mu \mathrm{m}$ \\
\hline$g_{\text {leak }}$ & $0.0006 \mathrm{~S} / \mathrm{cm}^{2}$ \\
\hline$g_{\mathrm{Na}}$ & $18 \mathrm{~S} / \mathrm{cm}^{2}$ \\
\hline$g_{\mathrm{LVA}}$ & $0.4 \mathrm{~S} / \mathrm{cm}^{2}$ \\
\hline$g_{\mathrm{HVA}}$ & $0.5 \mathrm{~S} / \mathrm{cm}^{2}$ \\
\hline
\end{tabular}

of spike threshold), hypothesized to result from $\mathrm{Na}^{+}$channel inactivation (Monsivais and Rubel, 2001).

We explored the relationship between membrane potential and spike threshold to better understand the biophysical mechanisms and limits of accommodation in NM. We used a two-step current-clamp protocol in which the first step depolarized the neuron to a steady state, followed by a second step that was increased in amplitude until a spike was evoked (Fig. 1D). This protocol was designed to mimic the effects of steady depolarizing inhibition, isolating the effects of voltage-dependent conductances while eliminating the shunting by the $\mathrm{GABA}_{\mathrm{A}}$ receptor conductance. Threshold was defined as the point at which the voltage trace shifted from a passive rise to an active response, indicated by an upward inflection point. We measured this empirically as the time point at which the second derivative of the voltage trace passed from negative to positive. The voltage traces in Figure $1 D$ are from a representative NM neuron exhibiting voltage threshold accommodation. In Figure 1 D1, a $2.0 \mathrm{nA}$ current step (top trace) evokes an action potential (bottom trace), and voltage threshold is labeled with an open arrowhead. In Figure $1 D 2$, an initial current step depolarizes the neuron to a steady state, during which a $2.0 \mathrm{nA}$ current step fails to evoke a spike (asterisk) and a rheobase is increased to $3.2 \mathrm{nA}$. The upward shift of voltage threshold (open arrowhead) indicates accommodation. In Figure 1D3, a greater initial depolarization (because of larger initial current step), causes an additional change in both rheobase and voltage threshold. In Figure $1 D 4$, the three action potentials of $D 1-D 3$ are shown on an expanded timescale to more clearly illustrate voltage threshold accommodation. Action poten- 
tials in NM neurons had relatively small amplitudes, particularly when evoked from a depolarized membrane potential. This is likely attributable to the distal axonal locus of spike generation (Kuba and Ohmori, 2009; Kuba et al., 2010). Voltage threshold exhibited an almost linear change as a function of membrane potential, indicating strong accommodation in these neurons across a broad range of depolarized membrane potentials (Fig. 1E, open symbols). Additionally, the difference between membrane potential and voltage threshold (Fig. $1 F$ ) increased slightly but significantly as NM neurons were depolarized $(n=13 ; p<$ 0.0001; one-way ANOVA), indicating that depolarization is efficient at inducing accommodation to such an extent that very large inputs are always needed to evoke spikes.

We also monitored the amount of current required to evoke a spike as a function of steady-state membrane potential. To avoid confusion with voltage threshold, we refer to "current threshold" as rheobase, defined as the amplitude of a square current pulse required to evoke a spike. The traces shown in Figure $1 D$ suggest that the amount of current required to evoke a spike at resting membrane potential was insufficient to reach threshold during a steady-state depolarization (Fig. $1 D 2, D 3$, asterisks). That is, more current is required to evoke a spike when the neuron is depolarized; depolarization has an inhibitory effect on these neurons. Rheobase for P3 NM neurons at rest was calculated to be $1.9 \pm 0.58 \mathrm{nA}$ (mean $\pm \mathrm{SD})$. Figure $1 G$ shows mean rheobase as a function of membrane potential. This function was judged to be nonlinear because a second-order regression accounted for more of the variance than a linear fit $\left(R^{2}=0.98\right.$ for second-order regression vs $R^{2}=0.92$ for a linear fit). This can be attributed to the fact that, during depolarization, input resistance is decreased (Fig. $1 C$ ), whereas spike threshold is "increased" (i.e., the difference between membrane potential and voltage threshold is greater) (Fig. $1 F)$.

\section{Modeling spike threshold changes in NM}

We used computational modeling to better understand the biophysical mechanisms underlying the spike threshold behavior of NM neurons during depolarization. Single-compartment models, in which all ion channels are contained within a spherical soma, can accurately replicate some of the spiking characteristics of the cochlear nuclei (Rothman and Manis, 2003). However, more recent work has shown that using a multicompartment model, which mimics the subcellular distribution of ion channels, provides a richer understanding of the behavior of cochlear nucleus neurons in response to a variety of stimulus paradigms. Elegant experiments, particularly by Kuba and Ohmori, have shown that segregation of $\mathrm{Na}^{+}$channels, and thus the locus of
B

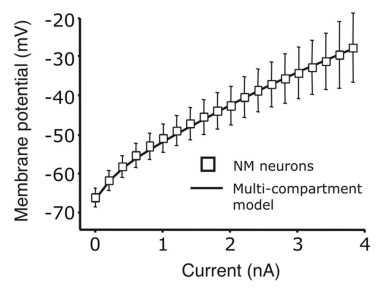

C

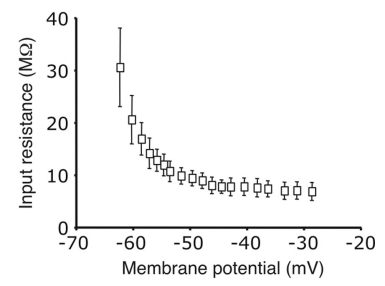

D4

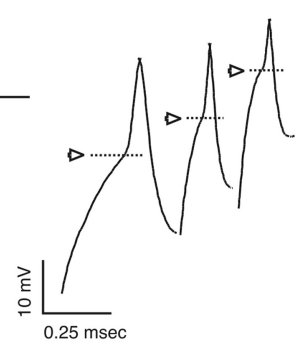

G

$\mathrm{F}$
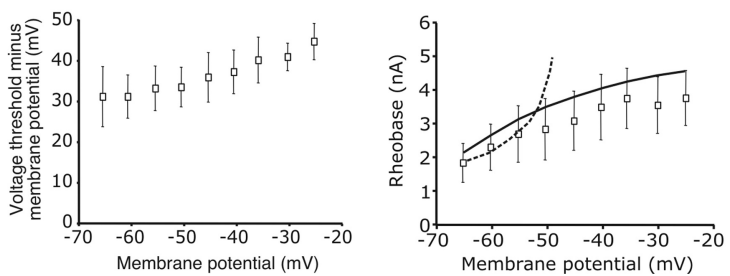

Figure 1. Action potential thresholds are highly flexible in NM neurons. $\boldsymbol{A}$, Depolarizing current steps (top) evoke single action potentials, followed by a steady-state depolarized potential in a representative P3 NM neuron. $\boldsymbol{B}$, Steady-state membrane poten(open squares), the multicompartment model (solid line) and the single-compartment model (dashed line). $\boldsymbol{F}$, The difference difference between rest and threshold across a broad range of membrane potentials. $\mathbf{G}$, Rheobase increases as a function of membrane potential, indicating decreased excitability during depolarization.

spike generation, away from the soma improves spike timing and high-frequency firing in NM and other auditory neurons (Kuba et al., 2006, 2010; Kuba and Ohmori, 2009). Our singlecompartment model replicated the spike threshold and VI function behavior of NM neurons in response to simple depolarizing current steps as in Figure $1 A-C$ (data not shown). However, when tested with the two-step current-clamp protocol of Figure $1 D$, spike threshold and rheobase (Fig. 1E, G, dashed lines) quickly deviated from that of NM neurons because of complete inactivation of $\mathrm{Na}^{+}$channels leading to depolarization block of spiking. Thus, we designed a multicompartment model of NM neurons similar to that of Kuba et al. (2006), with a soma containing mainly $\mathrm{K}^{+}$and leak channels, an initial segment of myelinated axon, a spike initiation zone containing a large concentration of $\mathrm{Na}^{+}$channels as well as $\mathrm{K}^{+}$and leak channels, and an extended axon consisting of alternating myelinated sections and nodes of Ranvier. Variables of our model were based on empirical measurements previously made in NM neurons and AVCN bushy cells (see Materials and Methods). We optimized 
A

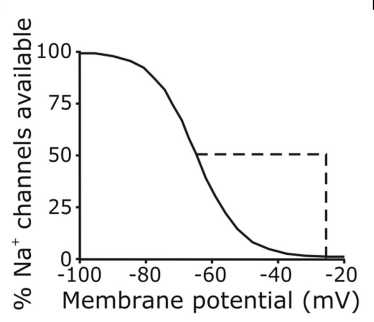

B

D

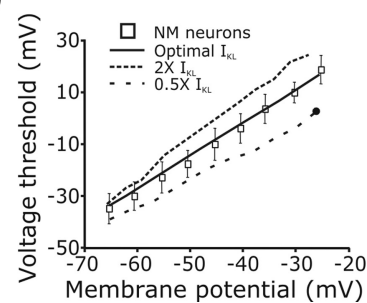

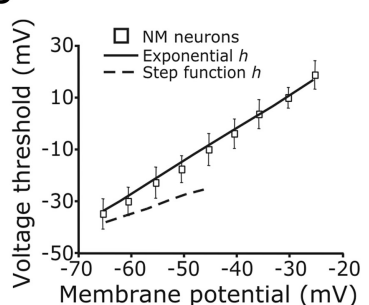

$\mathrm{E}$

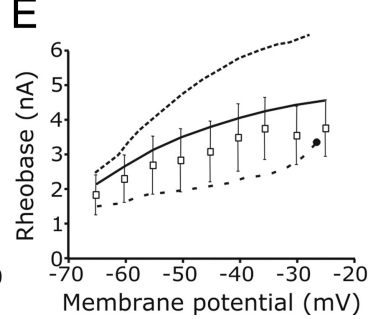

C

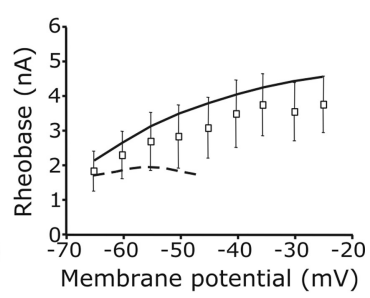

currents and smaller with lesser $\mathrm{K}^{+}$currents (Fig. 2 E). But again, the general pattern was not as severely disrupted as removal of $\mathrm{Na}^{+}$channel inactivation. These results indicate that both $\mathrm{Na}^{+}$ channel inactivation and $\mathrm{K}^{+}$currents are involved in threshold changes during depolarization: in general, $\mathrm{Na}^{+}$channel inactivation shapes the behavior of spike threshold during depolarization, whereas $\mathrm{K}^{+}$currents set the amplitude of these changes. This confirms the importance of both of these biophysical processes for depolarizing inhibition of NM neurons.

\section{NM neurons receive subthreshold EPSCs when input rate is high}

Unitary EPSCs are very large in mature $\mathrm{NM}$ neurons and it is unknown whether these inputs are generally suprathreshold or subthreshold in vivo. In a nondepressed state, that is, when stimulated at $1 \mathrm{~Hz}$, we calculated that unitary EPSC amplitude was $>30 \mathrm{nA}$ (based on amplitudes of $\sim 15 \mathrm{nA}$ in $\mathrm{NM}$ neurons voltage clamped at $-30 \mathrm{mV}$ ) (Brenowitz and Trussell, 2001). However, this synapse shows a large degree of synaptic depression (Brenowitz and Trussell, 2001). our model such that it reproduced the following behaviors as accurately as possible: the NM $V-I$ function (Fig. $1 B$, solid line in each figure), control spike threshold and accommodation (Fig. $1 E$ ), rheobase changes (Fig. $1 G$ ), as well as general spike shape and spike amplitude (data not shown).

A hypothesized mechanism underlying accommodation and inhibition in NM includes $\mathrm{Na}^{+}$channel inactivation by depolarizing IPSPs (dIPSPs) and $\mathrm{K}^{+}$channel activation (Monsivais and Rubel, 2001). Thus, we changed the biophysical properties of the multicompartment model to test the role of these processes in NM spike generation after depolarization to a steady state. To test the role of $\mathrm{Na}^{+}$channel inactivation, we changed the relationship between inactivation and voltage from the normal exponential process to a simple step function for depolarized potentials (Fig. $2 \mathrm{~A}$ ). As a result, the steady-state depolarization of the first step of the stimulus protocol caused no more $\mathrm{Na}^{+}$channel inactivation than was present at control membrane potential. When this was the case, the behavior of spike threshold (Fig. $2 B$ ) and rheobase (Fig. 2C) was dramatically altered as the model was depolarized. Additionally, when the model was depolarized beyond approximately $-48 \mathrm{mV}$, it began to fire bursts of spikes (data not shown), a behavior never seen in NM neurons. Thus, $\mathrm{Na}^{+}$channel inactivation plays a major role in setting spike threshold and changing rheobase as neurons are depolarized. This confirms the hypothesis that $\mathrm{Na}^{+}$channel inactivation is involved in changing threshold during depolarizing inhibition in NM.

We also changed the amplitude of low-voltage-activated $\mathrm{K}^{+}$ currents in our model to examine their effects on accommodation and rheobase shifts during depolarization. Doubling the amplitude of $\mathrm{K}^{+}$currents led to a slightly larger amount of accommodation, while halving $\mathrm{K}^{+}$currents decreased accommodation (Fig. 2D), but neither of these changes disrupted the general pattern of accommodation in the way $\mathrm{Na}^{+}$channel inactivation did. Changes to rheobase were larger with greater $\mathrm{K}^{+}$
Thus, the physiological range of excitatory inputs experienced by NM neurons is smaller than this maximal EPSC amplitude evoked at $1 \mathrm{~Hz}$. The mean in vivo spontaneous firing rate for eighth nerve fibers has been estimated to be $55-100 \mathrm{~Hz}$, and spike rates can exceed $400 \mathrm{~Hz}$ during sound stimulation (Fukui et al., 2006). It has been hypothesized that physiological firing rates in the eighth nerve will depress this synapse such that excitatory inputs to NM neurons will be mostly or entirely subthreshold, especially with moderate- or high-intensity acoustic stimulation (Reyes et al., 1994; Monsivais et al., 2000; Burger et al., 2005). Thus, NM neurons would have to act as monaural coincidence detectors, only spiking when they receive well timed inputs from multiple eighth nerve fibers. old in NM neurons and comparing these data with previous studies of EPSC depression during high-frequency stimulation. We developed a current-clamp stimulus using a simple $\alpha$ function with rise and decay kinetics (Fig. $3 A 1)\left(\tau_{\text {rise }}=0.15 \mathrm{~ms} ; \tau_{\text {decay }}=\right.$ $0.3 \mathrm{~ms}$ ) similar to real NM EPSCs (Brenowitz and Trussell, 2001). We varied the amplitude and measured the spike threshold of NM neurons to this stimulus ("EPSC threshold"). Additionally, we used conductance clamp input to measure the effect of depolarizing inhibition on EPSC threshold (Fig. 3A2). A longduration (onset $5 \mathrm{~ms}$ before the EPSC), square conductance input, with a reversal potential of $-37 \mathrm{mV}$, was used to mimic steady-state inhibition. ( $\mathrm{SD}, 0.50 \mathrm{nA} ; n=13$ ). Increasing depolarizing inhibitory conductances caused a significant increase in EPSC threshold (Fig. $3 B)(p<0.01$; one-way ANOVA). In Figure 3C, we illustrate a comparison between previously reported EPSC amplitudes [from Brenowitz and Trussell (2001); amplitudes were recorded at a holding potential of $-30 \mathrm{mV}$ in the original report, but we calculated what the amplitudes would be at a holding potential of
We sought to test this hypothesis by measuring EPSC thresh-

EPSC threshold in NM neurons was measured to be $2.86 \mathrm{nA}$ 
A1 A2

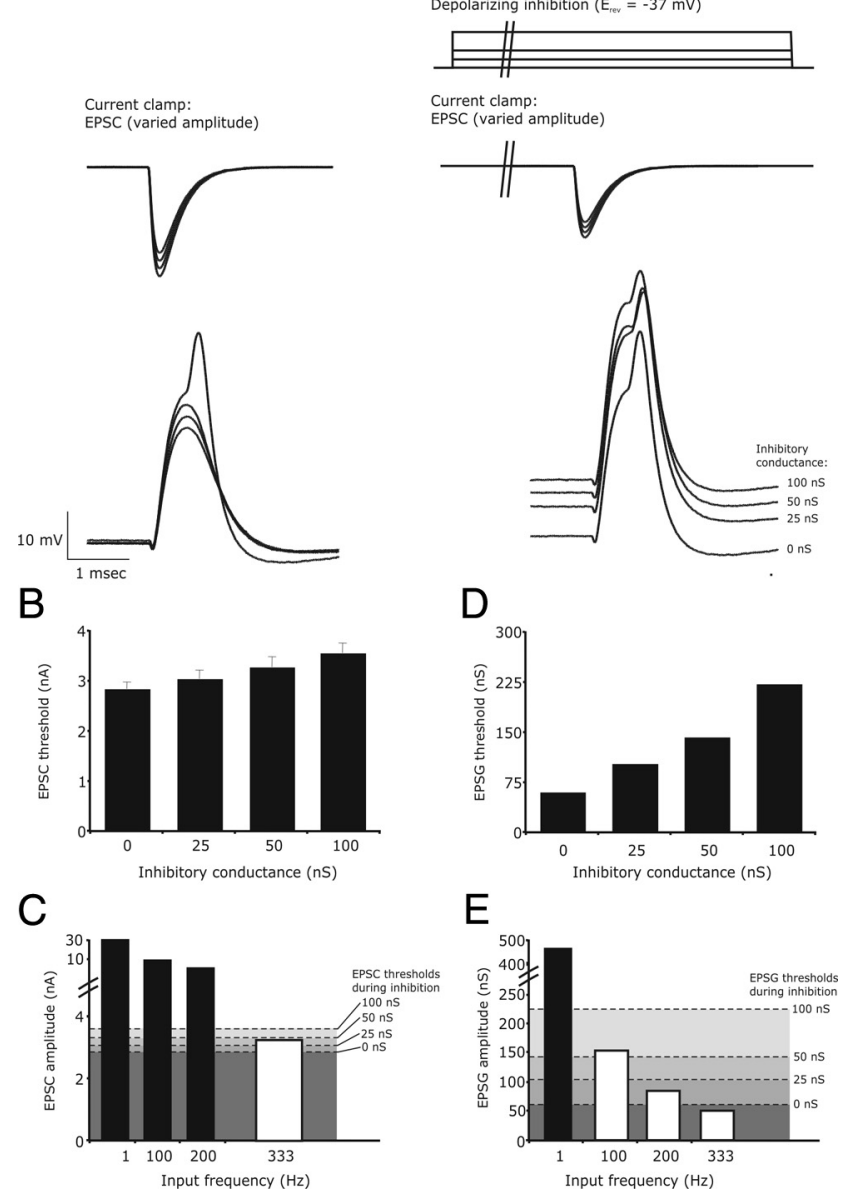

Figure 3. EPSCS can be subthreshold in NM under physiological conditions. $A$, Current and conductance clamp were used to stimulate NM neurons with realistic inputs. $\boldsymbol{A 1}$, Current-clamp "EPSCs" (top traces) were varied in amplitude until a spike was evoked (bottom traces) to measure EPSC threshold. A2, A conductance clamp input was used to mimic depolarizing inhibition. Square conductance steps had an onset $5 \mathrm{~ms}$ before the EPSC. The reversal potential for the conductance clamp input was $-37 \mathrm{mV}$. Spikes in response to just-threshold inputs show accommodation because of depolarizing inhibition. Voltage traces are offset because of depolarization by the conductance input. $\boldsymbol{B}$, EPSC threshold was increased by depolarizing conductances in neurons (mean \pm SEM; $n=13$ ). C, Bars show actual EPSC amplitude as a function of input frequency [from Brenowitz and Trussell (2001); amplitudes calculated for a membrane potential of $-65 \mathrm{mV}]$. EPSC thresholds from $\boldsymbol{B}$ are indicated by dashed lines. The shaded regions below each threshold indicate EPSC amplitudes that will be subthreshold during such inhibition. At high frequencies ( $333 \mathrm{~Hz}$ ), EPSC amplitude was subthreshold during inhibition. D, EPSG (reversal potential, $0 \mathrm{mV}$ ) threshold was measured in the multicompartment model under varying levels of depolarizing inhibitory conductance. EPSG threshold increased to a greater extent than EPSC threshold in $\boldsymbol{B}$. E, EPSC amplitudes shown in C were converted to conductance and illustrated as a function of input frequency. The dashed lines indicate the EPSG thresholds of the multicompartment model from $\boldsymbol{D}$. EPSGs become subthreshold at lower firing rates and lower levels of inhibition than EPSCS.

- $65 \mathrm{mV}$ for comparative purposes] with our measured EPSC thresholds (dashed lines). This comparison showed that EPSCs evoked at $200 \mathrm{~Hz}$ and slower are likely to be suprathreshold, whereas EPSCs evoked at higher frequencies (well within the physiological range) are much closer to threshold, becoming subthreshold during depolarizing inhibition.

To get a more precise measure of spike threshold in response to synaptic conductances, we measured the minimum excitatory postsynaptic conductance required to evoke a spike ("EPSG threshold"), using a similar protocol to that shown in Figure $3 A 2$.
The important distinction between these measures and the previously described EPSC threshold is that the reversal potential for EPSCs presented in current clamp is infinity, whereas the reversal potential for true synaptic conductances is $\sim 0 \mathrm{mV}$. Thus, as neurons are depolarized toward this reversal potential, synaptic inputs become much less effective at producing additional depolarization. The limitations of our conductance clamp system (see Materials and Methods) required us to make use of our multicompartment model for these measures. Our modeled data show that depolarizing inhibition caused substantial increase in EPSG threshold (Fig. 3D). This is logical in that depolarization induces voltage threshold accommodation, and as the changing voltage threshold approaches reversal potential, the excitatory conductance required to reach that threshold will increase exponentially. The differences between EPSC and EPSG threshold shifts during inhibition (Fig. 3, compare $B, D$ ) were striking. The $100 \mathrm{nS}$ of depolarizing inhibitory conductance increased EPSC threshold to $125 \%$ of the control value, whereas the same inhibition increased EPSG threshold to $375 \%$ of control.

We then compared EPSG thresholds to steady-state EPSG amplitudes recorded at varied eighth nerve spike frequencies [amplitudes calculated from EPSC amplitudes from Brenowitz and Trussell (2001)] (Fig. 3E). These comparisons show that eighth nerve inputs become subthreshold when axon spike rates reach $333 \mathrm{~Hz}$, in the absence of any inhibition. With the addition of modest levels of depolarizing inhibitory conductance, eighth nerve inputs become subthreshold at spike rates of $200 \mathrm{~Hz}$, and high levels of inhibition can result in subthreshold EPSGs even when afferents are firing at $100 \mathrm{~Hz}$. These measurements provide strong evidence that NM neurons receive subthreshold excitatory inputs under physiological conditions, such as when excitatory and inhibitory afferent input frequency increases during sound stimulation. Based on these data, we hypothesize that the large endbulb synapses of the eighth nerve evoke suprathreshold conductances in quiet conditions, whereas sound stimuli that drive more activity will shift inputs to subthreshold levels such that NM neurons require integration of multiple synaptic events to reach spike threshold. Importantly, eighth nerve spike rates can increase in response to both increases in sound frequency and intensity (Fukui et al., 2006). Thus, the shift of inputs from suprathreshold to subthreshold and the enhancement of temporal fidelity can increase the dynamic range of the system to sound intensities, emphasizing detection at low sound levels and temporal precision at higher sound levels, and can ensure accuracy of encoding phase information across a range of sound frequencies.

\section{Depolarizing inhibition narrows the coincidence detection window in NM}

Under conditions in which all synaptic events are subthreshold, neurons must temporally sum synaptic inputs to spike. Many auditory neurons have a narrow time window for integration, mostly because of fast EPSP kinetics and large voltage-dependent outward currents. In the auditory system, temporal integration is often quantified as the "coincidence detection window," defined as the time domain after an EPSP during which a second EPSP can easily sum to evoke a spike. Previous studies of NL neurons, the coincidence detector neurons that receive bilateral input from NM, have shown that depolarizing inhibition improves spike timing by narrowing the coincidence detection window (Funabiki et al., 1998). We examined the temporal integration in $\mathrm{NM}$ by measuring the width of the coincidence detection window under various levels of inhibition. As in the previous experiment, we first measured the EPSC threshold of each neuron. We then 
A

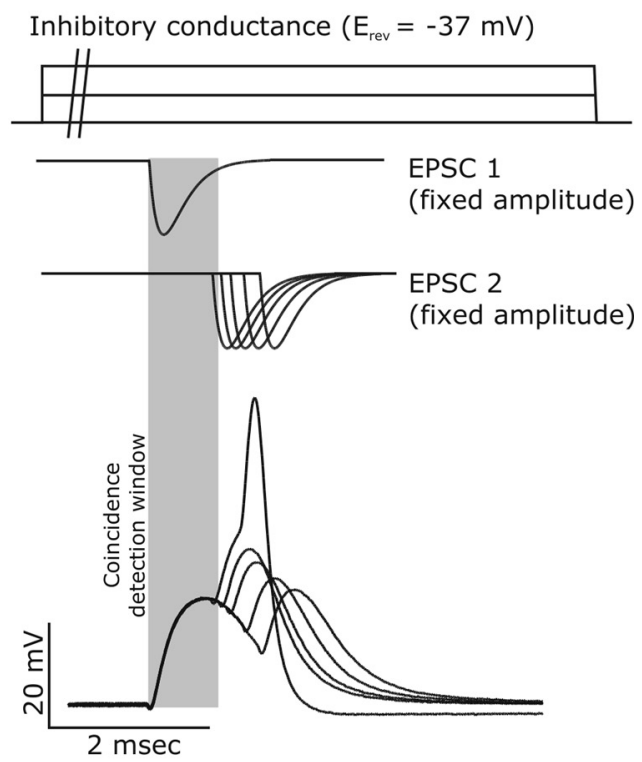

B

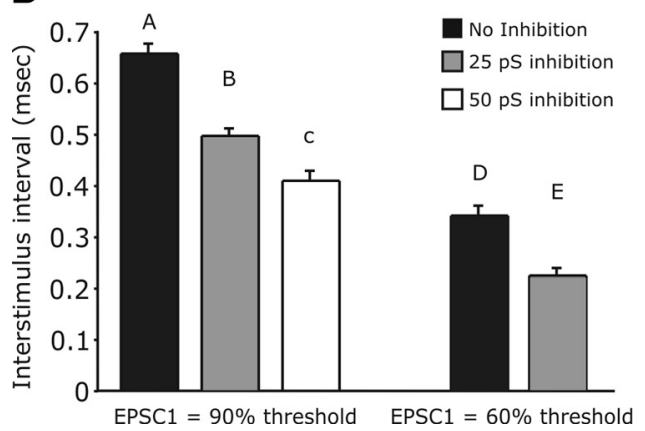

Figure 4. Depolarizing inhibition narrows the coincidence detection window. $\boldsymbol{A}$, NM neurons were stimulated with two EPSC waveforms in current clamp. EPSC amplitudes were set and interstimulus interval was decreased (middle traces). A second channel of the amplifier supplied depolarizing inhibition in conductance clamp (top traces). The bottom traces illustrate voltage responses of a representative neuron ( 0 inhibition). The coincidence detection window for this neuron is indicated by the shaded area. $\boldsymbol{B}$, The width of the coincidence detection window was significantly decreased by depolarizing inhibition in NM neurons (mean \pm SEM; $n=13$ ). Smaller EPSCS ( $60 \%$ of threshold) also had a significantly narrower coincidence summation window. Statistically distinct groupings are marked by letters above bars $(p<0.001$; two-way ANOVA, Bonferroni's post hoc tests).

set the amplitude of the first EPSC (EPSC1) to large (90\% of threshold) or small ( $60 \%$ of threshold). The interstimulus interval between EPSC1 and a second EPSC (EPSC2) of equal amplitude was varied and the coincidence detection window was then defined as the maximum interstimulus interval at which a spike was evoked (Fig. 4A). As above, we also used conductance clamp to provide depolarizing inhibition during the experiment.

Data from a representative NM neuron are illustrated in the bottom traces of Figure $4 \mathrm{~A}$ in response to this stimulus (EPSC amplitude, $90 \%$ threshold; no inhibition). Because of the fast kinetics of these neurons, the interstimulus interval between EPSCs had to be very brief for the resulting EPSPs to temporally sum and reach threshold. On average, the coincidence detection window for large EPSCs was $<0.7 \mathrm{~ms}$ (Fig. $4 B$ ). Depolarizing inhibition significantly decreased this value $(p<0.0001$; twoway ANOVA, Bonferroni's post hoc test). The coincidence detection window was much smaller when EPSC amplitudes were smaller (i.e., $60 \%$ of initial threshold rather than $90 \%$ ). This is as expected, because smaller inputs are briefer and thus have a more limited time period during with they can interact. This finding also reflects the idea that synaptic depression (i.e., decreasing input amplitude) enhances temporal fidelity in auditory neurons (Kuba et al., 2002; Cook et al., 2003). Again, depolarizing inhibition significantly narrowed the coincidence detection window for small EPSCs $(p<0.0001)$. True synaptic inputs, or synaptic inputs modeled with conductance clamp, would likely have an even narrower temporal integration window, particularly during depolarizing inhibition, because the depolarization induced by the first EPSP would bring membrane potential closer to the EPSP reversal potential, thus lessening the power of the second EPSP to further depolarize the neuron.

Additionally, we noted two interesting phenomena that these experiments were not directly designed to address. The first is that the EPSP that resulted from two inputs occurring just outside the temporal integration window did not appear to be twice the amplitude of a single input (as is apparent in the subthreshold traces of Fig. 4A), indicating the possibility that temporal summation in NM neurons was less than linear. Second, we noted that the integrated EPSP to these "poorly timed" inputs could exceed control voltage threshold, but did not evoke a spike, suggesting that subthreshold EPSPs could induce accommodation. We designed experiments to directly address these possibilities.

\section{Nonlinear temporal integration in NM neurons}

The physiological hallmarks of NM neurons (e.g., large leak and potassium currents, relatively small $\mathrm{Ca}^{2+}$ and NMDA currents) make them poor temporal integrators. Thus, we hypothesized that inputs occurring outside the coincidence detection window would sum in a less-than-linear fashion. Using a protocol similar to that shown in Figure 4A, we examined the peak amplitude of subthreshold EPSPs evoked at varied interstimulus intervals. The black traces in Figure $5 \mathrm{~A}$ illustrate the responses of a representative current-clamp experiment. To estimate linearity, we simply took the voltage waveform of a single control EPSP and added a second equal EPSP at each time point, and calculated the peak amplitude, producing our "linear summing model." These responses are illustrated in Figure $5 \mathrm{~A}$ by gray traces. Across a range of EPSC interstimulus intervals, the true voltage responses do not reach the same amplitude as the linear calculation, indicating less than linear summation. For group data, we normalized the actual EPSP amplitude to the expected (linear) amplitude, and plotted this value as a function of interstimulus interval (Fig. $5 B, C$ ). Note that the left-most point in these plots indicates the edge of the coincidence detection window, which changes depending on the level of inhibition. When inputs are large (90\% of threshold), they sum together very poorly outside the coincidence detection window, an effect that persists for up to $3 \mathrm{~ms}$ after EPSC1 (Fig. $5 B$ ). Summation between smaller EPSCs was closer to linear (Fig. $5 C$ ). Within the temporal integration window, summation is supralinear, with voltage-gated $\mathrm{Na}^{+}$channels activating and producing a spike. Outside this time domain, the less-than-linear summation is likely attributable to shunting of EPSC2 by voltagedependent $\mathrm{K}^{+}$channels activated by the first EPSP. Functionally, nonlinear summation will decrease the probability of asynchronous inputs evoking action potentials.

\section{Subthreshold inputs cause accommodation and EPSC threshold increases}

We sought to determine the extent of voltage threshold accommodation after a subthreshold excitatory input. To this end, we performed current-clamp experiments similar to those previ- 
ously described, but in which the amplitude of EPSC2 was varied until a spike was evoked (Fig. 6A). As previously, conductance clamp was used to produce a depolarizing inhibition. Traces in Figure 6A show recordings from a representative NM neuron under current clamp (with 0 nS inhibition), illustrating the radical changes in EPSC amplitude required to evoke a spike across time after an initial subthreshold input. This phenomenon is reflected in the mean data (Fig. $6 \mathrm{~B}$ ). The amplitude of EPSC1 was large, either 90 or $60 \%$ of threshold. Thus, when EPSC2 was given within the coincidence detection window (interstimulus interval, $<0.6 \mathrm{~ms}$ ), the input needed to reach threshold is relatively small. Note that in Figure $6 B$ the coincidence detection window is the time frame to the left of the point where the threshold functions cross the control threshold (dashed line). Outside of that window (interstimulus interval, greater than $\sim 0.6 \mathrm{~ms}$ ), however, in the same time domain in which temporal summation is less-than-linear, EPSC threshold to elicit a spike is greatly increased and does not return to control levels until interstimulus interval is $>3 \mathrm{~ms}$. The return of threshold to control levels is rapid enough that if afferents are firing at $333 \mathrm{~Hz}$, the response of the NM neuron will be unaffected for the next set of stimuli. This has additional importance during sound stimulation in that these threshold shifts will likely only affect responses within one cycle for lower frequency sounds.

This increase in EPSC threshold was smaller but still present when the initial EPSC was smaller in amplitude (Fig. 6C). Depolarizing inhibition, again presented as a square conductance input of 25 or $50 \mathrm{nS}$ with a reversal potential of $-37 \mathrm{mV}$, increased the amount of EPSC threshold change needed to evoke a spike, but not the duration of the effect.

To examine threshold accommodation, we measured voltage threshold in these experiments and show that subthreshold EPSCs induced a large depolarized shift in voltage threshold (Fig. 6D,E). As with the change in EPSC threshold, this effect is very strong just outside the coincidence detection window and returns to control values at around interstimulus intervals of $\sim 3 \mathrm{~ms}$. Importantly, voltage threshold approaches the reversal potential for excitation after a subthreshold excitatory input. This is particularly true during depolarizing inhibition, when the effect peaks with voltage threshold at $-10 \mathrm{mV}$. Because excitatory synaptic conductances have a reversal potential of $0 \mathrm{mV}$, the amplitude required to achieve threshold will increase exponentially during accommodation, and our currentclamp experiments again underestimated threshold changes. Thus, we measured changes to EPSG threshold after an initial subthreshold conductance using our multicompartment model (see Materials and Methods).

\section{Depolarizing inhibition produces a refractory period after subthreshold excitatory inputs}

We applied the same stimulus protocol illustrated in Figure $6 \mathrm{~A}$ to our multicompartment model, but used EPSGs with a reversal potential of $0 \mathrm{mV}$ in place of EPSCs. We then compared EPSG thresholds to physiological EPSGs amplitudes. Figure 7A illustrates EPSG threshold as a function of interstimulus interval after an initial subthreshold event (EPSG1, 90\% threshold). This function was similar in shape to the EPSC threshold recorded in NM neurons using current clamp (Fig. $6 \mathrm{~B}$ ). The time course of this function is slightly faster than EPSC threshold changes recorded in our in vitro experiments. This may be attributable in part to slight differences in kinetics between channel gating in the model and in real neurons, in part because of different experimental conditions across laboratories in which the measurements on which the model was based were made. We have included on this graph physiological NM EPSG amplitudes at various input frequencies (dashed lines; values shown in Fig. 3E). Note that the increase in EPSG threshold outside the coincidence detection window was large enough to render most depressed (highfrequency) inputs subthreshold. Thus, not only did spiking require very precise timing between multiple inputs, but equally or more important, the dramatic increase in threshold after a subthreshold input provides a mechanism by which asynchronous inputs were actively and profoundly excluded from evoking spikes.

The addition of depolarizing inhibition greatly increased EPSG threshold and enhanced the exclusion of spikes outside the coincidence detection window. Figure $7 B$ illustrates EPSG 
A
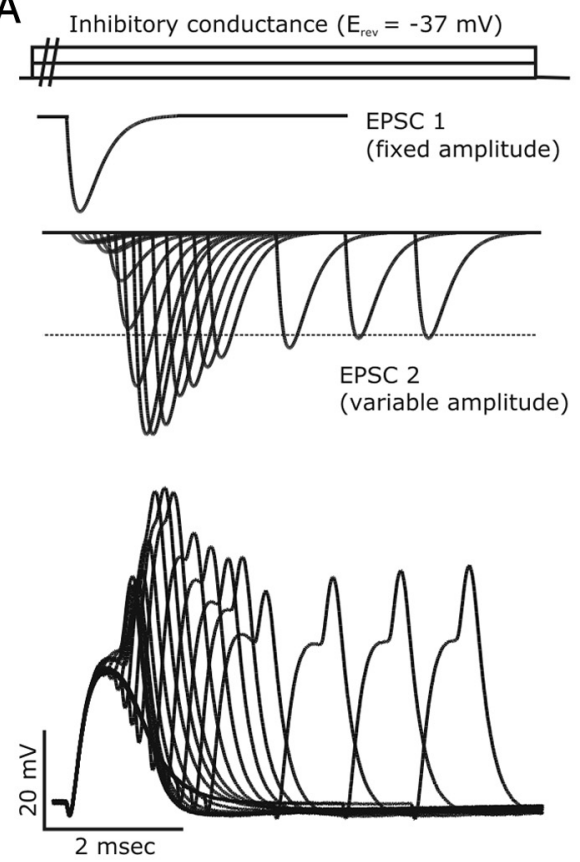

B

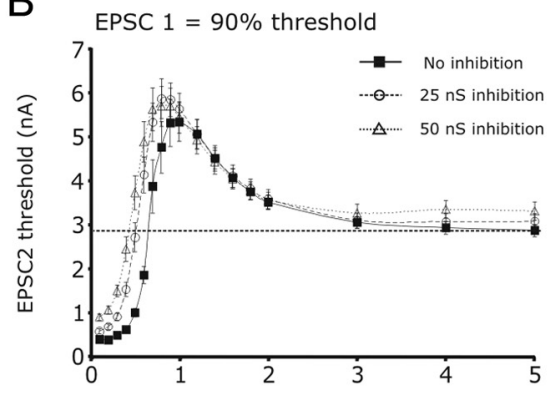

C

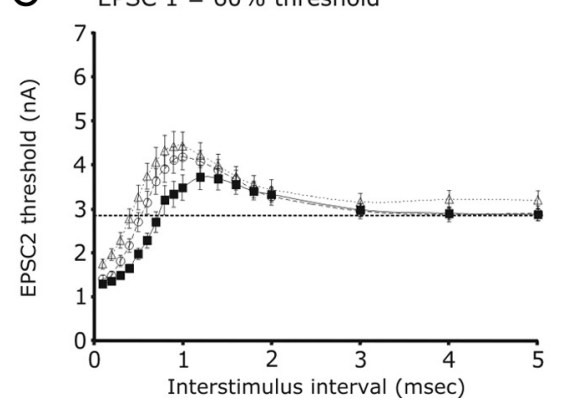

D

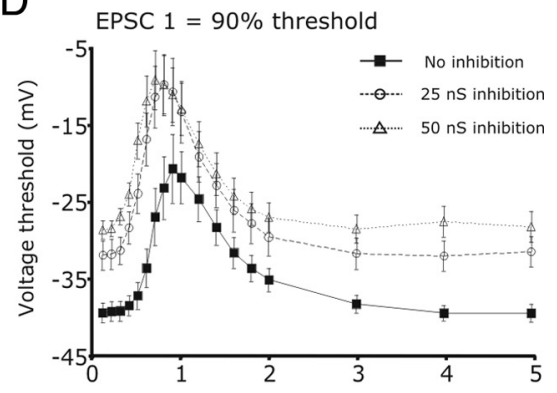

E

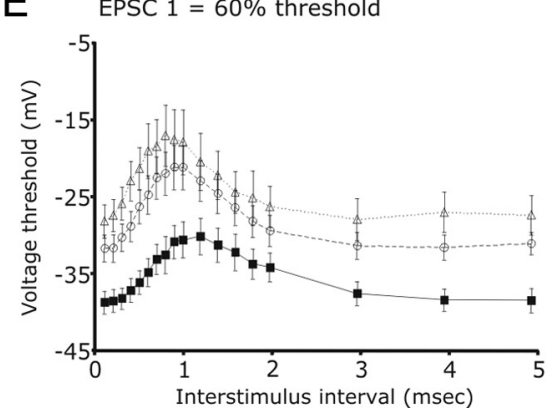

Figure 6. Subthreshold inputs increase EPSC threshold and cause accommodation. $\boldsymbol{A}$, The amplitude of EPSC1, modeled in current clamp, was fixed, whereas the amplitude of EPSC2 (middle traces) was varied at each interstimulus interval point until an action potential was evoked (bottom traces). Depolarizing inhibition was simulated with long-duration square pulses in conductance clamp, with a reversal potential of $-37 \mathrm{mV}$ (top traces). The horizontal dashed line in the middle traces over EPSC2 indicates control EPSC threshold. B, C, EPSC spike threshold was plotted as a function of interstimulus interval after an initial subthreshold input (EPSC1) for NM neurons ( $n=12$; mean \pm SEM). Control threshold is marked by a dashed line. Within the coincidence summation window (very small interstimulus intervals; less than $\sim 0.5 \mathrm{~ms}$ ), temporal summation was close to linear and EPSC2 spike threshold was low. Beyond the coincidence summation window EPSC2 spike threshold increased dramatically before returning to control levels after $\sim 3 \mathrm{~ms}$. Depolarizing inhibition enhanced this effect (open symbols). D, E, Voltage threshold accommodation was strong and followed the same function as ESPC2 spike threshold after a subthreshold input. Depolarizing inhibition also enhanced this effect.

A

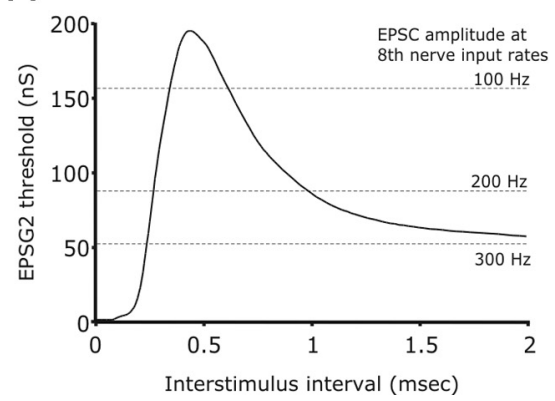

B

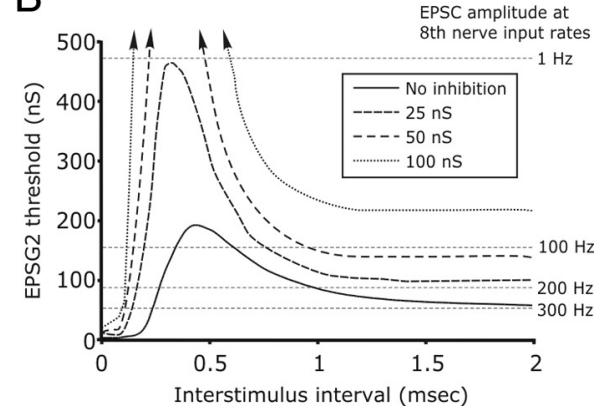

Figure 7. A refractory period follows subthreshold excitatory inputs. $A$, EPSG spike threshold was measured in the multicompartment model as a function of interstimulus interval, as in Figure 6 B. The initial input was set to $90 \%$ of threshold. Direct comparisons with real EPSG amplitudes at different input frequencies (dashed horizontal lines) show that threshold often rises well above physiological EPSG amplitudes when eighth nerve rate is elevated. $\boldsymbol{B}$, Depolarizing inhibition dramatically increased EPSG spike threshold in the multicompartment model. During a time period after the coincidence summation window, small inhibitory conductances raised spike generation threshold well above physiological EPSG amplitudes. During larger inhibitory conductances, spikes could not be evoked (between upward arrows), indicating an absolute refractory period despite the fact that no action potential was fired.

threshold as a function of interstimulus interval with the addition of 25,50 , and $100 \mathrm{nS}$ depolarizing inhibition (reversal potential, $-37 \mathrm{mV}$ ). These functions differed from those of Figure $6 \mathrm{~B}$ because of accommodation forcing voltage threshold to approach the reversal potential of the EPSGs. During the time period after the coincidence detection window, the lowest levels of inhibition elevated EPSG threshold to such an extent that only a completely non-depressed NM synapse (firing at $1 \mathrm{~Hz}$ ) could evoke a spike. Thus, under physiological conditions (given a mean spontaneous rate of $\sim 55-100 \mathrm{~Hz}$ for eighth nerve fibers), this time domain represents a virtual refractory period after a subthreshold input. Importantly, larger inhibitory conductances made this refractory period absolute, in that spikes were not evoked regardless of EPSG amplitude. Thus, the combined changes in threshold induced by depolarizing inhibition and subthreshold inputs produced a mechanism by which NM neurons completely exclude the possibility of spiking in response to asynchronous inputs.

\section{Discussion}

NM receives one to three large excitatory eighth nerve afferents per cell (Parks, 1981; Hackett et al., 1982). Mature NM neurons respond to GABAergic inhibition, from superior olivary nucleus (SON), with slow, depolarizing IPSPs that cause shunting and voltage threshold accommodation (Lachica et al., 1994; Hyson et al., 1995; Monsivais and Rubel, 2001). NM neuron spike trains are more temporally precise relative to sound phase than the eighth nerve (Fukui et al., 2006), and this precision depends on SON inhibition (Fukui et al., 2010). We sought to determine how excitatory and depolarizing inhibitory inputs are integrated in NM and the role of this integration in the improvement of spike timing between the eighth nerve and NM. We show that nonlinear summation between subthreshold EPSPs, voltage threshold accommodation, and shunting dramatically diminish the ability of asynchronous excitatory inputs to evoke a spike in 
NM. This is particularly profound during depolarizations such as those induced by GABAergic inhibition. When integrating subthreshold excitatory inputs and inhibitory input, the specific biophysical attributes of NM neurons can cause an absolute refractory period after subthreshold excitatory synaptic inputs. Figure $8 \mathrm{~A}$ illustrates this process, in which a sound stimulus evokes phase-locked spikes (vertical lines) in two eighth nerve axons, and a modeled NM neuron responds to these inputs. Large subthreshold inputs result in a brief refractory period, such that no spikes will be evoked by asynchronous inputs from other afferents. Importantly, our data show that such subthreshold refractory periods are relatively brief, and thus this refractoriness will not affect inputs during the next phase cycle of the stimulus. During a single subthreshold EPSP, the window of excitation is very brief, followed by a longer period during which excitability is dramatically diminished (Fig. $8 \mathrm{~B}$ ). These data lead us to hypothesize that the subthreshold refractory period helps NM neurons eliminate spiking in response to asynchronous subthreshold inputs and the elimination of errant spiking provides a mechanism for the observed increase in temporal fidelity of NM neurons relative to eighth nerve axons.

\section{Nonlinear temporal summation in NM}

Many of the synaptic and voltage-dependent adaptations of NM neurons promote less-than-linear temporal summation between EPSPs. First, voltage-dependent outward currents dominate over inward currents during depolarization (Koyano et al., 1996), reflected by the curvature of the $V-I$ function (Fig. $1 B$ ). Activation of the large low-voltage-activated $\mathrm{K}^{+}$conductances of NM (Reyes et al., 1994; Rathouz and Trussell, 1998) serves to keep EPSPs brief and decreases temporal jitter in action potential timing (Brenowitz and Trussell, 2001) and contributes to shunting by depolarizing inhibition (Howard et al., 2007). Activation of these conductances by subthreshold excitatory inputs contributes to the nonlinear temporal summation we observed. Mature NM neurons exhibit very large AMPA but small NMDA currents, also serving to keep EPSPs brief (Zhou and Parks, 1992; Lu and Trussell, 2007).

The morphology of neurons, including the location of synapses within the dendritic arbor and relative to one another, can also determine the mode of temporal summation (Carter et al., 2007; Jean-Xavier et al., 2007). Synapses in close proximity are more likely to be shunted by each other, whereas EPSPs evoked on different dendrites may sum linearly or supralinearly once the EPSPs have propagated to the soma. Mature NM neurons have either no or diminutive dendrites and both excitatory and inhibitory synapses are somatic (Parks and Rubel, 1978; Jhaveri and Morest, 1982; Carr and Boudreau, 1991, 1993). Thus, the synaptic arrangement and morphology of NM promotes both accurate spike timing and sublinear summation between EPSPs.

The linearity of temporal summation between EPSPs is strongly related to the relative timing between inputs (Margulis and Tang, 1998). Coincident EPSPs depolarize the membrane to a greater extent and thus have a greater probability of activating facilitating mechanisms, whereas non-coincident EPSPs are more susceptible to shunting by the voltage-dependent properties of the neuron activated by the first input (Margulis and Tang, 1998; Carter et al., 2007). Thus, neurons exhibiting linear or supralinear summation of coincident inputs may also have a second temporal window during which temporal summation is diminished. We show that, in NM neurons, there is a very brief coincidence detection window followed by a second time domain in which summation is profoundly less than linear, and in some
A
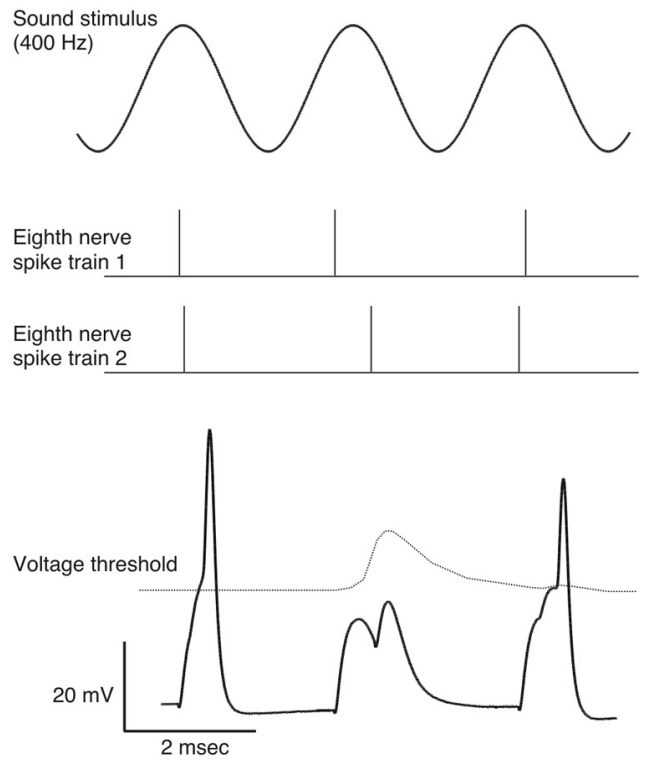

B
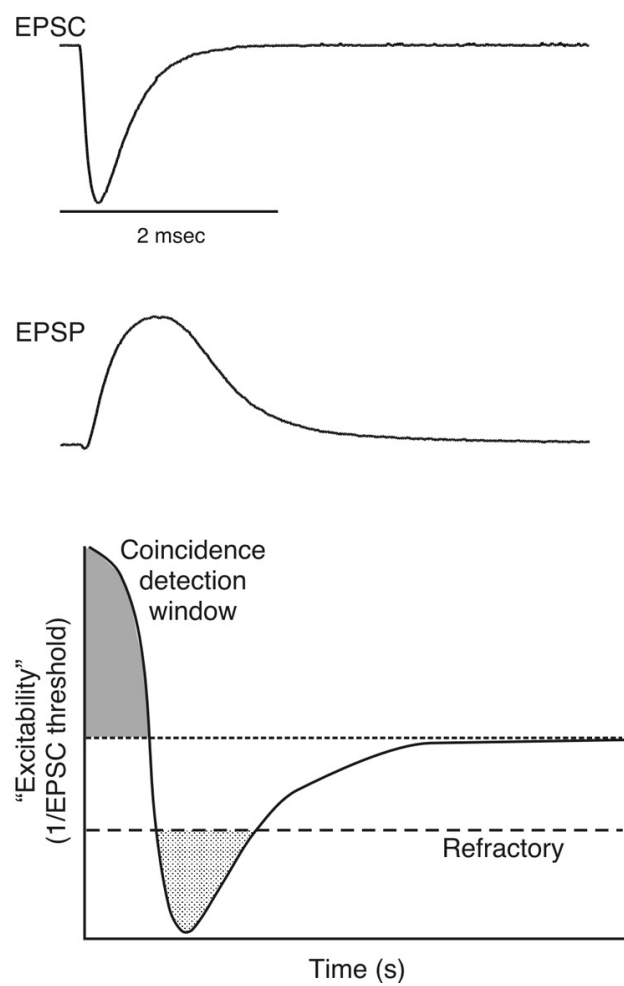

Figure 8. Elimination of poorly timed spikes in NM. A, A sound stimulus (top) evoked eighth nerve action potentials (middle, vertical lines) with slightly imperfect phaselocking. When the timing of eighth nerve action potentials is poor, as in the middle responses, the modeled neuron failed to initiate a spike (bottom). Accommodation of voltage threshold is shown by the dotted line. $\boldsymbol{B}, \mathrm{On}$ an expanded timescale, a single excitatory input (top) that evokes a subthreshold EPSP (middle) greatly changes the excitability of the neuron. This is represented as the inverse of EPSC threshold (bottom). At the very onset of the EPSP, during the coincidence detection window (gray shaded area), excitability is high. However, it very quickly drops well below control levels (dotted line) during the succeeding time period, decreasing to such an extent as to become refractory (dashed line and stippled area), after which it returns to control levels. 
cases refractory. We hypothesize that this second window is critically important for auditory function in that it greatly reduces spiking to asynchronous inputs, thereby enhancing phase locking.

\section{Mechanisms of accommodation and depolarizing inhibition}

The high intracellular $\mathrm{Cl}^{-}$concentration of mature NM neurons results in GABAergic depolarization (Hyson et al., 1995; Lu and Trussell, 2001; Monsivais and Rubel, 2001). Our data suggest that the synaptic conductance, activation of $\mathrm{K}^{+}$conductances, and inactivation of $\mathrm{Na}^{+}$channels all contributed to shunting and threshold accommodation, resulting in depolarizing inhibition in this system. Additionally, the anatomical restriction of $\mathrm{Na}^{+}$ channels to a spike initiation zone away from the soma appears key to maintaining excitability during dIPSPs. Our conductance clamp data show that depolarizing inhibition narrowed the coincidence detection window for subthreshold EPSPs. Such inhibition also enhanced the accommodation and EPSC threshold changes that occur during the non-coincident window after a subthreshold excitatory input such that non-coincident inputs become even more unlikely to evoke spikes.

During depolarizing inhibition, subthreshold EPSPs temporally sum more poorly than under control conditions. Shunting by inhibitory postsynaptic conductances diminishes temporal summation between EPSPs, particularly in systems in which GABA acts as a nonpolarizing or depolarizing neurotransmitter (Graham and Redman, 1994; Banke and McBain, 2006). Activation of outward currents and inactivation of inward currents by subthreshold dIPSPs will also decrease temporal summation (Edwards, 1990a,b). Voltage-activated $\mathrm{K}^{+}$currents play an integral role in determining how GABAergic inputs are integrated in the avian auditory brainstem (Lu and Trussell, 2001; Monsivais and Rubel, 2001; Howard et al., 2007). In other systems exhibiting depolarizing inhibition, both the relative timing between and relative location of excitatory and inhibitory synapses can influence how excitatory and depolarizing inhibitory inputs integrate (Gulledge and Stuart, 2003; Jean-Xavier et al., 2007). In NM, the somatic location of both inhibitory and excitatory synapses (Parks, 1981; Jhaveri and Morest, 1982; Code et al., 1989; Carr and Boudreau, 1991) heightens the power of dIPSP shunting, thus augmenting the inhibitory nature of these inputs.

Inactivation of $\mathrm{Na}^{+}$channels by subthreshold depolarizations is a key component of depolarizing inhibition in some other systems (Eccles et al., 1961; French et al., 2006). This mechanism can decrease supralinear summation by reducing $\mathrm{Na}^{+}$channelmediated EPSP facilitation (Margulis and Tang, 1998). dIPSPs decrease NM excitability through spike threshold accommodation (Monsivais and Rubel, 2001). Our modeling data further support the conclusion that $\mathrm{Na}^{+}$channel inactivation plays an important role in this accommodation.

\section{Threshold changes and the refractory period may improve spike timing}

Temporal fidelity can be increased across a synapse in a system in which neurons receive only a small number of suprathreshold inputs (Xu-Friedman and Regehr, 2005b). This depends, in part, on asynchronous inputs occurring during the refractory period, after an action potential evoked by an initial, well timed input. We expect this to be the case in NM neurons when input rates for both excitatory and inhibitory synapses are low compared with maximal firing rates.

Our data strongly suggest that, as afferent nerve firing rates increase, NM EPSPs shift from suprathreshold to subthreshold.
Subthreshold synapses can also improve spike timing: after multiple inputs sum to evoke a spike, the refractory period suppresses additional spikes in response to late inputs ( $\mathrm{Xu}$-Friedman and Regehr, 2005a). However, improvement of timing by these mechanisms assumes that the earliest inputs received are well timed relative to the stimulus and that lagging inputs are poorly timed, and responses to these lagging inputs are eliminated. Like the work by Xu-Friedman and Regehr, our data show that NM neurons require highly synchronized subthreshold EPSPs to reach threshold. However, we show that timing may be improved by elimination of spiking in response to early subthreshold inputs, in that a refractory period is induced and spiking within that phase cycle of the stimulus will be blocked. This implies that spike timing is improved by the active elimination of responses to poorly timed activity, both early and lagging. NM exhibits lower maximal firing rates and better phase locking compared with the eighth nerve (Fukui et al., 2006). Eighth nerve spike rates increase with both the frequency and the intensity of sound stimuli, so subthreshold refractory periods may play a role in the enhancing temporal information regarding both of these properties of auditory stimuli. Finally, depolarizing inhibition is highly effective at enhancing such a mechanism because it induces threshold accommodation and lowers input resistance in ways that hyperpolarizing or nonpolarizing inhibitions do not. Depolarizing inhibition plays an important role in shifting excitation from suprathreshold to subthreshold and inducing subthreshold refractory periods, thus eliminating spikes to asynchronous inputs and enhancing temporal fidelity. Indeed, blockade of the inhibition of NM by SON increases firing rates and degrades phase locking during sound stimulation, highlighting the importance of this input in auditory processing (Fukui et al., 2010).

\section{References}

Banke TG, McBain CJ (2006) GABAergic input onto CA3 hippocampal interneurons remains shunting throughout development. J Neurosci 26:11720-11725.

Belluzzi O, Sacchi O, Wanke E (1985) A fast transient outward current in the rat sympathetic neurons studied under voltage-clamp conditions. J Physiol 358:91-108.

Brenowitz S, Trussell LO (2001) Maturation of synaptic transmission at end-bulb synapses of the cochlear nucleus. J Neurosci 21:9487-9498.

Burger RM, Cramer KS, Pfeiffer JD, Rubel EW (2005) Avian superior olivary nucleus provides divergent inhibitory input to parallel auditory pathways. J Comp Neurol 481:6-18.

Carr CE, Boudreau RE (1991) Central projections of auditory nerve fibers in the barn owl. J Comp Neurol 314:306-318.

Carr CE, Boudreau RE (1993) Organization of the nucleus magnocellularis and the nucleus laminaris in the barn owl: encoding and measuring interaural time differences. J Comp Neurol 334:337-355.

Carter AG, Soler-Llavina GJ, Sabatini BL (2007) Timing and location of synaptic inputs determine modes of subthreshold integration in striatal medium spiny neurons. J Neurosci 27:8967-8977.

Code RA, Burd GD, Rubel EW (1989) Development of GABA immunoreactivity in brainstem auditory nuclei of the chick: ontogeny of gradients in terminal staining. J Comp Neurol 284:504-518.

Cook DL, Schwindt PC, Grande LA, Spain WJ (2003) Synaptic depression in the localization of sound. Nature 421:66-70.

Costa PF (1996) The kinetic parameters of sodium currents in maturing acutely isolated rat hippocampal CA1 neurones. Brain Res Dev Brain Res 91:29-40.

Dasika VK, White JA, Carney LH, Colburn HS (2005) Effects of inhibitory feedback in a network model of avian brain stem. J Neurophysiol 94:400-414.

Eccles JC, Eccles RM, Magni F (1961) Central inhibitory action attributable to presynaptic depolarization produced by muscle afferent volleys. J Physiol 159:147-166.

Edwards DH (1990a) Mechanisms of depolarizing inhibition at the crayfish giant motor synapse. I. Electrophysiology. J Neurophysiol 64:532-540. 
Edwards DH (1990b) Mechanisms of depolarizing inhibition at the crayfish giant motor synapse. II. Quantitative reconstruction. J Neurophysiol 64:541-550.

French AS, Panek I, Torkkeli PH (2006) Shunting versus inactivation: simulation of GABAergic inhibition in spider mechanoreceptors suggests that either is sufficient. Neurosci Res 55:189-196.

Fukui I, Ohmori H (2004) Tonotopic gradients of membrane and synaptic properties for neurons of the chicken nucleus magnocellularis. J Neurosci 24:7514-7523.

Fukui I, Sato T, Ohmori H (2006) Improvement of phase information at low sound frequency in nucleus magnocellularis of the chicken. J Neurophysiol 96:633-641.

Fukui I, Burger RM, Ohmori H, Rubel EW (2010) GABAergic inhibition sharpens the frequency tuning and enhances phase locking in chicken nucleus magnocellularis neurons. J Neurosci 30:12075-12083.

Funabiki K, Koyano K, Ohmori H (1998) The role of GABAergic inputs for coincidence detection in the neurones of nucleus laminaris of the chick. J Physiol 508:851-869.

Graham B, Redman S (1994) A simulation of action potentials in synaptic boutons during presynaptic inhibition. J Neurophysiol 71:538-549.

Gulledge AT, Stuart GJ (2003) Excitatory actions of GABA in the cortex. Neuron 37:299-309.

Hackett JT, Jackson H, Rubel EW (1982) Synaptic excitation of the second and third order auditory neurons in the avian brain stem. Neuroscience 7:1455-1469.

Hossain WA, Antic SD, Yang Y, Rasband MN, Morest DK (2005) Where is the spike generator of the cochlear nerve? Voltage-gated sodium channels in the mouse cochlea. J Neurosci 25:6857-6868.

Howard MA, Burger RM, Rubel EW (2007) A developmental switch to GABAergic inhibition dependent on increases in Kv1-type $\mathrm{K}^{+}$currents. J Neurosci 27:2112-2123.

Hyson RL, Reyes AD, Rubel EW (1995) A depolarizing inhibitory response to GABA in brainstem auditory neurons of the chick. Brain Res 677:117-126.

Jackson H, Hackett JT, Rubel EW (1982) Organization and development of brain stem auditory nuclei in the chick: ontogeny of postsynaptic responses. J Comp Neurol 210:80-86.

Jean-Xavier C, Mentis GZ, O’Donovan MJ, Cattaert D, Vinay L (2007) Dual personality of GABA/glycine-mediated depolarizations in immature spinal cord. Proc Natl Acad Sci U S A 104:11477-11482.

Jhaveri S, Morest DK (1982) Sequential alterations of neuronal architecture in nucleus magnocellularis of the developing chicken: a Golgi study. Neuroscience 7:837-853.

Koyano K, Funabiki K, Ohmori H (1996) Voltage-gated ionic currents and their roles in timing coding in auditory neurons of the nucleus magnocellularis of the chick. Neurosci Res 26:29-45.

Kuba H, Ohmori H (2009) Roles of axonal sodium channels in precise auditory time coding at nucleus magnocellularis of the chick. J Physiol 587:87-100.

Kuba H, Koyano K, Ohmori H (2002) Synaptic depression improves coincidence detection in the nucleus laminaris in brainstem slices of the chick embryo. Eur J Neurosci 15:984-990.

Kuba H, Ishii TM, Ohmori H (2006) Axonal site of spike initiation enhances auditory coincidence detection. Nature 444:1069-1072.

Kuba H, Oichi Y, Ohmori H (2010) Presynaptic activity regulates $\mathrm{Na}^{+}$ channel distribution at the axon initial segment. Nature 465:1075-1078.

Lachica EA, Rübsamen R, Rubel EW (1994) GABAergic terminals in nu- cleus magnocellularis and laminaris originate from the superior olivary nucleus. J Comp Neurol 348:403-418.

Lu T, Trussell LO (2001) Mixed excitatory and inhibitory GABA-mediated transmission in chick cochlear nucleus. J Physiol 535:125-131.

Lu T, Trussell LO (2007) Development and elimination of endbulb synapses in the chick cochlear nucleus. J Neurosci 27:808-817.

Margulis M, Tang CM (1998) Temporal integration can readily switch between sublinear and supralinear summation. J Neurophysiol 79:2809_ 2813.

Monsivais P, Rubel EW (2001) Accommodation enhances depolarizing inhibition in central neurons. J Neurosci 21:7823-7830.

Monsivais P, Yang L, Rubel EW (2000) GABAergic inhibition in nucleus magnocellularis: implications for phase locking in the avian auditory brainstem. J Neurosci 20:2954-2963.

Oertel D (1999) The role of timing in the brain stem auditory nuclei of vertebrates. Annu Rev Physiol 61:497-519.

Parks TN (1981) Morphology of axosomatic endings in an avian cochlear nucleus: nucleus magnocellularis of the chicken. J Comp Neurol 203:425-440.

Parks TN, Rubel EW (1978) Organization and development of the brain stem auditory nuclei of the chicken: primary afferent projections. J Comp Neurol 180:439-448.

Rathouz M, Trussell L (1998) Characterization of outward currents in neurons of the avian nucleus magnocellularis. J Neurophysiol 80:2824-2835.

Reyes AD, Rubel EW, Spain WJ (1994) Membrane properties underlying the firing of neurons in the avian cochlear nucleus. J Neurosci 14:5352-5364.

Rothman JS, Manis PB (2003) The roles potassium currents play in regulating the electrical activity of ventral cochlear nucleus neurons. J Neurophysiol 89:3097-3113.

Rubel EW, Smith DJ, Miller LC (1976) Organization and development of brain stem auditory nuclei of the chicken: ontogeny of n. magnocellularis and n. laminaris. J Comp Neurol 166:469-489.

Scott LL, Mathews PJ, Golding NL (2005) Posthearing developmental refinement of temporal processing in principal neurons of the medial superior olive. J Neurosci 25:7887-7895.

Tang ZQ, Gao H, Lu Y (2009) Control of a depolarizing GABAergic input in an auditory coincidence detection circuit. J Neurophysiol 102:1672-1683.

Xu-Friedman MA, Regehr WG (2005a) Dynamic-clamp analysis of the effects of convergence on spike timing. I. Many synaptic inputs. J Neurophysiol 94:2512-2525.

Xu-Friedman MA, Regehr WG (2005b) Dynamic-clamp analysis of the effects of convergence on spike timing. II. Few synaptic inputs. J Neurophysiol 94:2526-2534.

Yang L, Monsivais P, Rubel EW (1999) The superior olivary nucleus and its influence on nucleus laminaris: a source of inhibitory feedback for coincidence detection in the avian auditory brainstem. J Neurosci 19:2313-2325.

Zhang S, Trussell LO (1994a) A characterization of excitatory postsynaptic potentials in the avian nucleus magnocellularis. J Neurophysiol 72:705-718.

Zhang S, Trussell LO (1994b) Voltage clamp analysis of excitatory synaptic transmission in the avian nucleus magnocellularis. J Physiol 480:123-136.

Zhou N, Parks TN (1992) Developmental changes in the effects of drugs acting on NMDA or non-NMDA receptors on synaptic transmission in the chick cochlear nucleus (nuc. magnocellularis). Brain Res Dev Brain Res 67:145-152. 